\title{
Análisis molecular de la resistencia a fluoroquinolonas y macrólidos en aislados de Campylobacter jejuni de humanos, bovinos y carne de ave
}

\author{
Gisela González-Hein, Ninoska Cordero, Patricia García y Guillermo Figueroa
}

\section{Molecular analysis of fluoroquinolones and macrolides resistance in Campylobacter jejuni isolates from humans, bovine and chicken meat}

Background: Campylobacter sp.- one of the leading causes of bacterial food-borne gastrointestinal illness worldwide- is increasingly resistant to fluoroquinolone and macrolide antimicrobials, which has become a major concern for public health. Objective: To describe the susceptibility patterns of Campylobacter jejuni strains to erythromycin and ciprofloxacin and to explore the origin of its resistance in human isolates. Material and Method: In this study, fifty-five ciprofloxacin and erythromycin susceptibility patterns of $C$. jejuni strains isolated from humans with diarrheal disease, performed by broth microdilution MIC, were compared with 55 and 44 isolates from chicken meat and bovines respectively, obtained from the Metropolitan Region, Chile. Results: Of the 55 human isolates of C. jejuni, 33 (60\%) were resistant to ciprofloxacin and all were sensitive to erythromycin. Of the 55 isolates from chicken meat, $32(58.2 \%)$ were resistant to ciprofloxacin and $1.8 \%$ were resistant to erythromycin. Of the 44 isolates of $C$. jejuni from cattle, $8(18.2 \%)$ were resistant to ciprofloxacin and all were sensitive to erythromycin. Four PFGE patterns matched with certain resistance profiles and grouped isolates from human and animal. Conclusion: The findings showed continued effectiveness of erythromycin for campylobacteriosis and a high percentage of $C$. jejuni strains ciprofloxacin-resistant. This is interesting because it is considered that the presence of ciprofloxacin resistant strains in broiler meat can be in part the source of resistance to this antimicrobial in humans.

Key words: Campylobacter jejuni; chicken; bovine, human; resistance, ciprofloxacin, erythromicin.

Palabras clave: Campylobacter jejuni; pollos, bovinos, humanos, resistencia, ciprofloxacina, eritromicina.

\section{Introducción}

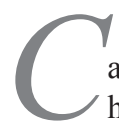

ampylobacter jejuni es uno de los principales agentes causales de gastroenteritis bacteriana en humanos $^{1-3}$. La campilobacteriosis por C. jejuni se asocia al consumo de alimentos o agua contaminada, siendo los pollos, pavos, bovinos y ovinos los principales reservorios ${ }^{1,4}$. A pesar que la campilobacteriosis humana es, por lo general, una enfermedad autolimitada, la terapia antimicrobiana es necesaria en casos graves y prolonga$\operatorname{dos}^{5}$. En la actualidad, macrólidos y fluoroquinolonas son considerados los antimicrobianos de primera y segunda elección, respectivamente, en el tratamiento de la campilobacteriosis. Sin embargo, en los años recientes, la emergencia de resistencia a estos grupos de antimicrobianos ha complicado el tratamiento empírico de la campilobacteriosis $^{6,7}$. En los últimos años, se produjo un incremento en la resistencia del C. jejuni a fluoroquinolonas en aislados de $C$. jejuni en humanos, alcanzando frecuencias de 32,4 y $46,6 \%$ en Chile ${ }^{8-10}$, situación que también se ha dado en otros países ${ }^{11-13}$. En el ámbito nacional, fluoroquinolonas y macrólidos están autorizados para su uso en medicina veterinaria, de acuerdo con el registro de Medicamentos Veterinarios del Servicio Agrícola y Ganadero ${ }^{14}$ y también se usan en medicina humana. Se sabe también que en el país, la cantidad de antimicrobianos del grupo de las quinolonas y fluoroquinolonas destinada para uso en medicina veterinaria es muy superior al que se usa en medicina humana ${ }^{15}$.

La determinación precisa de la susceptibilidad in vitro de Campylobacter sp., es de vital importancia para garantizar una terapia antimicrobiana adecuada de los pacientes con formas graves de la enfermedad y, también, para el monitoreo eficaz de la resistencia a los antimicrobianos en Campylobacter spp. a nivel mundial ${ }^{7}$. Los estudios de susceptibilidad antimicrobiana en aislados de Campylobacter spp., descritos por el Instituto de Estandarización Clínico y de Laboratorio (Clinical and Laboratory Standards Institute-CLSI) han precisado los puntos de corte y estandarizado los métodos de dilución en agar y dilución en caldo ${ }^{16-18}$. Otros métodos como el E-test ${ }^{\circledR}$ han dado resultados comparables y reproducibles ${ }^{19}$, no así la difusión en agar por disco ${ }^{7}$.

Un elemento crucial en el reconocimiento y control de
Universidad de Chile.

Instituto de Nutrición y Tecnología de Alimentos-INTA. Laboratorio de Microbiología y Probióticos (GGH, NC, GF).

Pontificia Universidad Católica de Chile.

Escuela de Medicina. Departamento de Laboratorios Clínicos. Laboratorio de Microbiología (PG).

Declaramos no tener conflictos de interés.

Financiamiento: Beca para Estudio de Doctorado en Chile, CONICYT y Beca para Estudio de Doctorado en Chile, CONICYT.

Recibido: 12 de noviembre de 2012 Aceptado: 4 de febrero de 2013

Correspondencia a: Gisela González-Hein gighein@yahoo.es 
enfermedades es la capacidad de analizar los patógenos aislados de humanos y de identificar las fuentes probables de contaminación hasta llegar al reservorio y vías de transmisión. Para realizar estos análisis se requieren metodologías de biología molecular que permiten establecer las similitudes genéticas entre las bacterias aisladas. En este contexto, los Centros para el Control de las Enfermedades y Prevención, de Estados Unidos de América- E.U.A., han desarrollado una red nacional de sub-tipificación molecular para la vigilancia de las enfermedades transmitidas por los alimentos, llamada PulseNet. Ésta se ha expandido a otras regiones del mundo como Canadá, Asia, África, Europa, Medio Oriente, América Latina y el Caribe y se ha basado para la sub-tipificación de las bacterias patógenas en la técnica de la electroforesis en gel de campo pulsado (EGCP) que consiste en la migración de fragmentos de $\mathrm{ADN}$ a través del gel de agarosa, como consecuencia de un cambio constante del campo eléctrico durante la electroforesis ${ }^{20}$.

Con los objetivos de determinar los patrones de susceptibilidad in vitro a ciprofloxacina y eritromicina (antimicrobianos de importancia crítica humana, de acuerdo a la Organización Mundial de la Salud ${ }^{21}$ ) y de conocer el origen de la resistencia en los aislados humanos de nuestra área geográfica (Región Metropolitana-RM), se compararon aislados de humanos con enterocolitis con los provenientes de carne de pollo y de bovinos que proveían a la región, mediante su caracterización fenotípica y molecular.

\section{Material y Método}

El presente estudio, retrospectivo, consistió en un análisis microbiológico y molecular de cepas de C.jejuni aisladas de humanos, pollos y bovinos.

Cepas bacterianas: se estudiaron 55 cepas de C.jejuni provenientes de pacientes con cuadros diarreicos (43 cepas de pacientes pediátricos $\leq 14$ años de edad y 12 , de pacientes $>14$ años), 44 de hisopados rectales de bovinos y 55 de carne de pollo industrial aisladas entre los años 2006 y 2010 en la R.M. Las cepas fueron recuperadas de cepario en agar Skirrow con el factor de crecimiento $\mathrm{FBP}^{22}$ e incubadas en microaerofilia a $42^{\circ} \mathrm{C}$ por $48 \mathrm{~h}$. La confirmación de las cepas de C. jejuni se realizó por las características de la colonia, características a la tinción, hidrólisis de hipurato y por la prueba de reacción de polimerasa en cadena (RPC) que amplifica el gen potencial de la oxido-reductasa ${ }^{23}$.

Estudio de susceptibilidad in vitro: se realizó mediante la determinación de la concentración inhibitoria mínima (CIM) por el método de microdilución en caldo acorde a las recomendaciones del CLSI ${ }^{16-18}$. En breve, se usaron microplacas estériles de 96 pocillos con fondo cónico y con tapas y como medio, caldo Mueller Hinton ajustado de cationes y suplementado con sangre de caballo lisada
$(2,5 \%)^{16}$. Se prepararon inóculos de los aislados de $C$. jejuni equivalentes a $0,5 \mathrm{McF}$ arland para luego llevarlos a una concentración en el pocillo de $5 \times 10^{5} \mathrm{ufc} / \mathrm{ml}$ siguiendo los procedimientos descritos en el documento M07-A $8^{18}$. El volumen del inóculo en cada pocillo fue de $10 \mu \mathrm{L}$. Los antimicrobianos evaluados fueron eritromicina y ciprofloxacina, ensayándose concentraciones entre 1 y $512 \mu \mathrm{g} / \mathrm{mL}$ y 1 y $128 \mu \mathrm{g} / \mathrm{mL}$, respectivamente. En todas las microplacas se usó además C. jejuni ATCC ${ }^{\circledR} 33560$ como cepa control en la determinación de la CIM. Se incluyeron, además, pocillos controles de esterilidad (medio sin inoculo, ni antimicrobiano) y un pocillo control de multiplicación de cada aislado (medio sin antimicrobiano más el inoculo de la bacteria). Las microplacas fueron incubadas por $48 \mathrm{~h}$ a $37^{\circ} \mathrm{C}$ en microaerofilia. Los puntos de corte que se utilizaron corresponden a los descritos para este patógeno en el documento M45A, del CLSI, 2006 (Tabla 1$)^{16}$.

Análisis estadístico. Posteriormente se usó la prueba de $\chi^{2}$ o Fisher, para comparar las proporciones de aislados resistentes según origen. Dado que la prueba $\chi^{2}$ requiere que las frecuencias esperadas en cada celdilla no sean demasiado bajas, al obtener frecuencias esperadas menores de 5, en las tablas de contingencias 2 × 2, se usó la prueba de Fisher ${ }^{24}$.

Estudio molecular de C. jejuni: para caracterizar los patrones de macrorestricción de los aislados de C. jejuni de diversos orígenes en función a su patrón de resistencia, se usó EGCP con la enzima SmaI acorde a la metodología recomendada por PulseNet ${ }^{25}$. El revelado de los patrones de macrorestricción se realizó por corrida en gel de agarosa con bromuro de etidio y transiluminación en UV. Se interpretó a los aislados con pulsotipos o patrones EGCP SmaI diferentes cuando diferían en una banda ${ }^{26}$.

\section{Resultados}

De los 55 aislados de $C$. jejuni de humanos, 33 (60\%) presentaron resistencia a ciprofloxacina y todos presentaron sensibilidad a eritromicina. De los 55 aislados procedentes de carne de pollo, 32 (58,2\%) presentaron resistencia a ciprofloxacina y un aislado resultó resistente a eritromicina (1,8\%). De los 44 aislados de C. jejuni de bovinos, ocho $(18,2 \%)$ presentaron resistencia a ciprofloxacina y todos resultaron sensibles a eritromicina. Sólo una de las 154 cepas evaluadas presentó resistencia a ambos antimicrobianos (Tabla 2).

La proporción de aislados resistentes a ciprofloxacina varió según su origen $(\mathrm{p}<0,05)$, siendo menor en bovinos. La presencia de aislados resistentes a eritromicina fue independiente del origen del aislado $(\mathrm{p}>0,05)$.

Se obtuvieron 93 diferentes patrones EGCP SmaI ${ }^{25}$, de los cuales 11 patrones mantuvieron aislados con los 
Tabla 1. Puntos de corte para los antimicrobianos ensayados (M45A CLSI, 2006)

\begin{tabular}{|lccc|} 
Antimicrobiano & $\begin{array}{c}\text { Sensible } \\
(\boldsymbol{\mu} \mathrm{g} / \mathrm{ml})\end{array}$ & $\begin{array}{c}\text { Intermedio } \\
(\boldsymbol{\mu g} / \mathrm{ml})\end{array}$ & $\begin{array}{c}\text { Resistente } \\
(\boldsymbol{\mu} \mathrm{g} / \mathrm{ml})\end{array}$ \\
Eritromicina & $\leq 8$ & 16 & $\geq 32$ \\
Ciprofloxacina & $\leq 1$ & 2 & $\geq 4$ \\
\hline
\end{tabular}

mismos perfiles de resistencia y CIMs. De los 11 patrones EGCP, tres compartieron aislados de origen humano y de pollos y uno compartió aislados de origen bovino y humano (Figura 1).

\section{Discusión}

La proporción de cepas resistentes a fluoroquinolonas ha aumentado rápidamente ${ }^{27}$ ocasionando un problema mundial en el tratamiento de la enteritis en general y particularmente por Campylobacter $\mathrm{spp}^{11}$. En Argentina, Japón y países europeos, el porcentaje de resistencia a quinolonas en humanos bordea o supera el $60 \%{ }^{11-13}$. Las frecuencias altas y muy similares $(p>0,05)$ de resistencia a ciprofloxacina encontradas en el presente estudio en aislados de humanos $(60 \%)$ y de pollos broiler $(58,2 \%)$ son concordantes a las reportadas en países de Europa y Argentina y parecen ser consistentes con la opinión científica muy difundida que las aves de corral son la fuente más importante de campilobacteriosis humana por $C$. jejuni $i^{12}$. Variadas publicaciones han asociado el aumento de la resistencia de Campylobacter spp., a fluoroquinolonas con la introducción de estos fármacos en la industria avícola, principalmente de enrofloxacina, cuyo metabolito activo es ciprofloxacina ${ }^{8,12,28-30}$. Países como Australia no han permitido el uso de fluoroquinolonas en producción animal destinada al consumo y reportan una baja resistencia a fluoroquinolonas $(3,2 \%)$ en aislados de Campylobacter de origen humano, donde los casos de aislados resistentes fueron atribuidos a viajes ${ }^{31}$. En Dinamarca, el empleo de fluoroquinolonas en la producción de pollos fue restringido. En el año 2002, el uso de fluoroquinolonas en la producción de pollos broiler decreció significativamente y ese año, acorde con el Programa Danés Integrado de Vigilancia e Investigación de Resistencia Antimicrobiana (DANMAP) no fueron aisladas cepas resistentes a quinolonas en pollos broiler ${ }^{32}$.

En la presente investigación, en humanos, la resistencia a ciprofloxacina se encontró en aislados principalmente de pacientes pediátricos $(73 \%)$ en los cuales no se recomienda usar las fluoroquinolonas como tratamiento empírico de diarrea. Dada esta situación, la probabilidad de adquisición de este tipo de resistencia intra-tratamiento es escasa, y es más probable que la infección fuese provocada por cepas de $C$. jejuni ya resistentes.
Tabla 2. Susceptibilidad antimicrobiana de cepas de Campylobacter jejuni de diverso origen aisladas en la Región Metropolitana de Chile (n: 154)

\begin{tabular}{lcccccc}
$\begin{array}{l}\text { Origen } \\
\text { aislado }\end{array}$ & $\begin{array}{c}\text { Sensible } \\
\mathbf{n}(\%)\end{array}$ & $\begin{array}{c}\text { Ciprofloxacina } \\
\text { Intermedio } \\
\mathbf{n}(\%)\end{array}$ & $\begin{array}{c}\text { Resistente } \\
\mathbf{n}(\%)\end{array}$ & $\begin{array}{c}\text { Sensible } \\
\mathbf{n}(\%)\end{array}$ & $\begin{array}{c}\text { Eritromicina }^{\mathrm{b}} \\
\text { Intermedio } \\
\mathbf{n}(\%)\end{array}$ & $\begin{array}{c}\text { Resistente } \\
\mathbf{n}(\%)\end{array}$ \\
$\begin{array}{l}\text { Humano } \\
\text { (n: } 55)\end{array}$ & $21(38,2)$ & $1(1,8)$ & $33(60)$ & $55(100)$ & 0 & 0 \\
$\begin{array}{l}\text { Pollo } \\
\text { (n: } 55)\end{array}$ & $23(41,8)$ & 0 & $32(58,2)$ & $54(98,2)$ & 0 & $1(1,8)$ \\
$\begin{array}{l}\text { Bovino } \\
\text { (n: } 44)\end{array}$ & $36(81,8)$ & 0 & $8(18,2)$ & $44(100)$ & 0 & 0 \\
\hline
\end{tabular}

a) Las proporciones de aislados resistentes a ciprofloxacina variaron según origen $(p<0,05)$. b) No diferencias significativas en la proporción de aislados resistentes a eritromicina al comparar humanos versus animales domésticos (pollos más bovinos), p > 0,05.

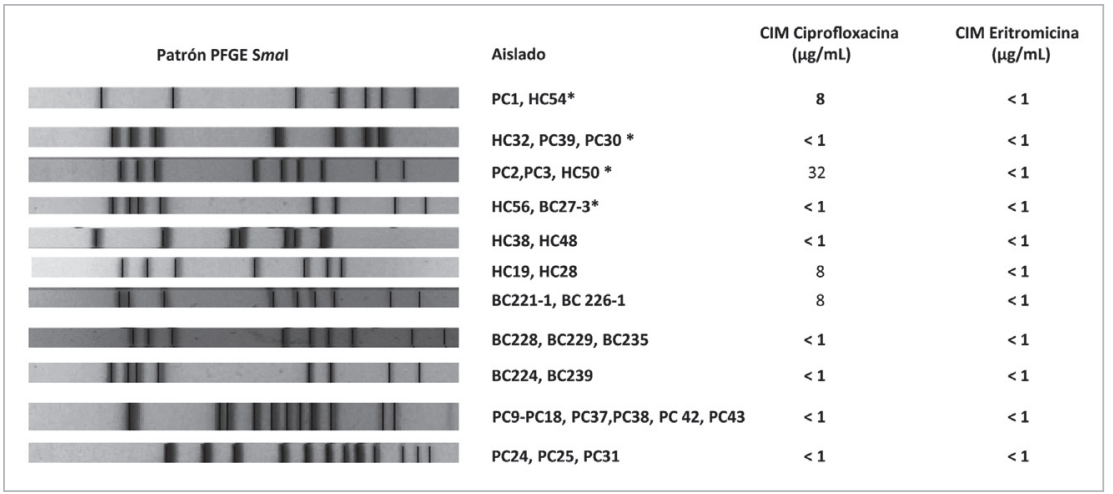

Figura 1. Aislados de C. jejuni de orígenes diversos que se caracterizaron por compartir los mismos patrones EGCP Smal y concentraciones inhibitorias mínimas para ciprofloxacina y eritromicina. EGCP: electroforesis en gel de campo pulsado. PC: aislados de pollo. HC: aislados humanos. BC: aislados de bovinos. *Que comparten aislados de origen humano y animal. Los patrones EGCP Smal, fueron enviados a publicación a la revista Cyta J Food, doi: 10.1080/19476337.2012.722564²6.

En contraste, las frecuencias de resistencia en cepas de origen humano a eritromicina son bajas y concordantes también con las de países europeos y las reportadas previamente en Chile ${ }^{8-9}$. Esto podría explicarse por el hecho que la selección de resistencia a macrólidos durante el tratamiento con este grupo de antimicrobianos es infrecuente ${ }^{32}$. Sin embargo, ya que los macrólidos se usan en medicina humana y frecuentemente en medicina veterinaria $^{21}$, especialmente en reservorios de Campylobacter spp., existe un riesgo de emergencia de resistencia. Lamentablemente, están aumentando las frecuencias de resistencia a macrólidos en varios países, lo que para E.U.A., Europa y Taiwán comienza a ser un problema de salud pública mayor ${ }^{34}$.

En el presente estudio, la presencia de aislados resistentes puede deberse probablemente a la participación de uno o más de los diversos mecanismos responsables de resistencia descritos para las fluoroquinolonas y los macrólidos en Campylobacter spp. Referente a los meca- 
nismos genéticos responsables de resistencia detectados en $C$. jejuni se ha reportado que la presión selectiva de fluoroquinolonas conduce rápidamente a resistencia antibacteriana $^{29}$. En C. jejuni la resistencia a fluoroquinolonas se genera por mutaciones puntuales en la región determinante de resistencia a quinolonas del gen gyrA. La bomba de eflujo CmeABC también se ha sido identificada como un sistema de resistencia a fluoroquinolonas ${ }^{30}$ y la sobre-expresión del gen $m f d$ (que codifica para la proteína Mfd que participa en el mecanismo de reparación del ADN en el proceso de la transcripción), podría incrementar la aparición espontánea de mutantes resistentes a fluoroquinolonas ${ }^{35}$. Para la resistencia a eritromicina, se describe en Campylobacter spp., una mutación cromosomal que genera una modificación en el sitio blanco del ribosoma (A2075 $\rightarrow$ G del gen 23S ARNr). La bomba de eflujo CmeABC actuaría sinérgicamente con las mutaciones de la zona 23S ARNr aumentando drásticamente las CIMs para eritromicina y tilosina en Campylobacter coli ${ }^{36}$.

La relación génica estrecha entre aislados de $C$. jejuni de origen humano y de origen animal (carne de pollo y bovinos) fue ya reportada en el país por nuestro equipo de investigación ${ }^{26}$. Al analizar los patrones de EGCP SmaI generados ${ }^{26}$ con los resultados de susceptibilidad antimicrobiana del presente estudio, observamos que ciertos patrones de EGCP, indistinguibles entre ellos, coincidieron también en sus fenotipos de resistencia y que algunos de ellos compartieron aislados de origen humano y animal. En otros países, ya se ha mostrado que ciertos genotipos de C. jejuni se han relacionado con determinados patrones de susceptibilidad antimicrobiana ${ }^{37,38}$ y que los mismos genotipos de $C$. jejuni circulan entre hombre $\mathrm{y}$ animales domésticos ${ }^{4,39}$.

Las similitudes encontradas en las tasas de resistencia a antimicrobianos, como también la detección de los mismos pulsotipos y patrones de resistencia en aislados de $C$. jejuni de origen humano y de pollos broiler, sugieren que estos últimos podrían actuar como una importante fuente de contagio para los humanos en nuestro país. Es requerida mayor investigación sobre otras fuentes potenciales de campilobacteriosis en el país como también la inclusión de $C$. jejuni en evaluaciones de riesgo, considerando especialmente la matriz alimentaria, carne de ave.

Los resultados del presente estudio enfatizan además en la trascendencia de mantener una vigilancia rigurosa de la resistencia a antimicrobianos de importancia crítica humana, en cepas de $C$. jejuni de origen humano como también en los aislados de origen animal (especialmente en pollo broiler) y del ambiente.

Agradecimientos. A Juan Carlos Román del Laboratorio de Microbiología, Escuela de Medicina, de la Pontificia Universidad Católica de Chile y a Miriam Troncoso del Laboratorio de Microbiología y Probióticos del INTA, Universidad de Chile quienes colaboraron en la presente investigación y a las Becas Apoyo Realización Tesis Doctoral, CONICYT y Becas para Estudio de Doctorado en Chile, CONICYT.

\section{Resumen}

Introducción: El incremento de la resistencia en Campylobacter sp. (una de las principales causas de gastroenteritis bacteriana de origen alimentario) a fluoroquinolonas y macrólidos, es un problema en salud pública. Objetivo: Conocer los patrones de susceptibilidad in vitro de Campylobacter jejuni a eritromicina y ciprofloxacina y conocer el origen de su resistencia en aislados de humanos. Material y Método: En este estudio, se compararon las susceptibilidades a ciprofloxacina y eritromicina-CIM efectuadas por microdilución en caldo- de 55 aislados de C. jejuni provenientes de humanos con enterocolitis, con 55 aislados de carne de pollo y 44 de bovinos obtenidos en la Región Metropolitana, Chile. Resultados: De 55 aislados de C. jejuni de humanos, 33(60\%) presentaron resistencia a ciprofloxacina y todos presentaron susceptibilidad a eritromicina. De 55 aislados procedentes de carne de pollo, $32(58,2 \%)$ presentaron resistencia a ciprofloxacina y un aislado resultó resistente a eritromicina $(1,8 \%)$. De 44 aislados de bovinos, $8(18,2 \%)$ presentaron resistencia a ciprofloxacina y todos resultaron sensibles a eritromicina. Cuatro patrones de electroforesis a campo pulsado coincidieron en sus perfiles de resistencia y agruparon aislados de origen humano y animal. Conclusiones: Los resultados muestran que eritromicina continúa siendo efectiva para el tratamiento de la campilobacteriosis y que existe un alto porcentaje de cepas resistentes a ciprofloxacina. Se considera probable que la presencia de cepas resistentes a ciprofloxacina en la carne de pollo puede ser en parte el origen de la resistencia a este fármaco en humanos.

\section{Referencias bibliográficas}

1.- European Food Safety Authority, European Centre for Disease Prevention and Control. The European Union Summary Report on Trends and Sources of Zoonoses, Zoonotic Agents and Food-borne Outbreaks in 2010 EFSA J 2012; 10: 2597-3039.

2.- European Food Safety Authority.
Scientific opinion on Campylobacter in broiler meat production: control options and performance objectives and/or targets at different stages of the food chain. EFSA J 2011; 9: 2105-246.

3.- Centers for Disease Control and Prevention. CDC estimates of foodborne illness in United States. En: http://www.cdc.gov/ foodborneburden/2011-foodborne-estimates. html (consultado el 23-09-2012).

4.- Wilson D J, Gabriel E, Leatherbarrow A J, Cheesbrough J, Gee S, Bolton E, et al. Tracing the source of campylobacteriosis. En: http:// www.ncbi.nlm.nih.gov/pubmed/18818764 (consultado el 23-09-2012).

5.- Allos BM. Campylobacter jejuni infections: update on emerging issues and trends. Clin Infect Dis 2001; 32: 1201-6. 
6.- Aarestrup FM, Engberg J. Antimicrobial resistance of thermophilic Campylobacter. Vet Res 2001; 32: 311-21.

7.- Lehtopolku M, Kotilainen P, Puukka P, Nakari U, Siitonen A, Eerola E. Inaccuracy of the disk diffusion method compared with the agar dilution method for susceptibility testing of Campylobacter spp. J Clin Microbiol 2012; 50: 52-6.

8.- García P C, Valenzuela N S, Rodríguez V L, León E C, Fernández H J. Susceptibilidad antimicrobiana de Campylobacter jejuni aislado de coprocultivos en Santiago de Chile. Rev Chilena Infectol 2009; 26: 511-4.

9.- Rivera N, Bustos R, Montenegro S, Sandoval M, Castillo J L, Fernández H, et al. Genotipificación y resistencia antibacteriana de cepas de Campylobacter spp. aisladas en niños y en aves de corral. Rev Chilena Infectol 2011; 28: 555-62.

10.- Flores R, Duery O, Valenzuela M, Ronda E. Identificación y susceptibilidad antimicrobiana de aislados humanos de Campylobacter jejuni y Campylobacter coli desde el año 2008 hasta primer trimestre 2011. X Jornadas Científicas Instituto de Salud Pública 2011. En: http:// www.ispch.cl/documentos/libro_xjc_v2012.pdf (consultado el 23-09-2012).

11.- Notario R, Borda N, Gambandé T, Bermejo J, Ponessa A, Toledo V. Cepas de Campylobacter jejuni resistentes a quinolonas aisladas de humanos, gallinas y pollos. Medicina (Buenos Aires) 2011; 71: 331-5.

12.- European Food Safety Authority. The European Union Summary report on antimicrobial resistance in zoonotic and indicator bacteria from humans, animals and food in 2010. EFSA J 2012; 10: 2598.

13.- Senok A, Yousif A, Mazi W, Sharaf E, Bindayna K, Elnima el-A, et al. Pattern of antibiotic susceptibility in Campylobacter jejuni isolates of human and poultry origin. Jpn J Infect Dis 2007; 60: 1-4.

14.- Chile, Servicio Agrícola Ganadero. Medicamentos Veterinarios. En: http:// www2.sag.gob.cl/pecuaria/medicamentos/ medicamentos_list.asp (consultado el: 23-09-2012).

15.- Millano A, Barrientos M, Gómez C, Tomova A, Bushmann H, Dolz H, et al. Uso inadecuado y excesivo de antibióticos: Salud pública y salmonicultura en Chile. Rev Med Chile 2011; 139: 107-18.

16.- Clinical and Laboratory Standards Institute. Methods for antimicrobial dilution and disk susceptibility testing of infrequently isolated or fastidious bacteria: Approved guideline. CLSI document M45-A. Clinical and Laboratory Standards Institute 2006; 26: 16-39.

17.- Clinical and Laboratory Standards Institute. Performance standards for antimicrobial susceptibility testing; Nineteenth informational supplement. CLSI document M100-S19. Clinical and Laboratory Standards Institute 2009; 29: 112-3.

18.- Clinical and Laboratory Standards Institute. Methods for dilution antimicrobial susceptibility tests for bacteria that grow aerobically approved standard-Eight Edition. CLSI document M07-A8. Clinical and Laboratory Standards Institute 2009; 29: 9-49.

19.- Rennie R P, Turnbull L, Brosnikoff C, Cloke J. First comprehensive evaluation of the M.I.C. evaluator device compared to Etest and CLSI reference dilution methods for clinical strains of anaerobes and other antimicrobial susceptibility testing of fastidious bacterial species. J Clin Microbiol 2012; 50: 1153-7.

20.- Normandin AM. Red PULSE NET en America Latina para Vigilancia de Enfermedades Transmitidas por Alimentos. En: http://epi. minsal.cl/epi/html/elvigia/vigia19/VIGIA1909. pdf (consultado el 21-01-2013).

21.- OMS (Organización Mundial de la Salud). Critically important antimicrobials for human medicine. En: http://www.who.int/foodsafety/ foodborne_disease/CIA_2nd_rev_2009.pdf (consultado el 23-09-2012).

22.- Tangwatcharin $P$, Chanthachum $S$, Khopaibool P, Chambers, JR y Griffiths, M.W. Media for the aerobic resuscitation of Campylobacter jejuni. J Food Prot 2007; 70: 1099-109.

23.- Cloak OM, Fratamico PM. A multiplex polymerase chain reaction for the differentiation of Campylobacter jejuni and Campylobacter coli from a swine processing facility and characterization of isolates by Pulse-Field Gel Electrophoresis and antibiotic resistance profile. J Food Prot 2002; 65: 266-73.

24.- Siegel S. Capítulo 6: El caso de dos muestras independientes. Siegel S, editor. Diseño experimental no paramétrico aplicado a las ciencias de la conducta, 1 ed. México: Editorial F. Trillas, S.A; 1970, p. 120-88

25.- Pulsenet, CDC. One-Day (24-26 h) standardized laboratory protocol for molecular subtyping of Campylobacter jejuni by pulsed field gel electrophoresis (PFGE), 2009. En: http://www. pulsenetinternational.org/ (consultado el 23-092012).

26.- González-Hein G, García P, Foerster C, Troncoso M, Figueroa G. Campylobacter jejuni isolated from human cases in Chile showed indistinguishable PFGE profiles with strains isolated from poultry and bovine sources. En: http://www.tandfonline.com/doi/abs/10.1080/1 9476337.2012.722564 (consultado el 14-012013).

27.- Engberg J, Aarestrup F, Taylor D, Gerner-Smidt P, Nachamkin I. Quinolone and macrolide resistance in Campylobacter jejuni and Campylobacter coli: resistance mechanisms and trends in human isolates. Emerg Infect Dis 2001; 7: 24-34

28.- Vanhoof R, Gossens H, Coignau H, Stas G, Butzler J. Susceptibility pattern of Campylobacter jejuni from human and animal origins to different antimicrobial agents. Antimicrob Agents Chemother 1982; 21: 990-2.

29.- Threlfall E. Fluoroquinolone-resistant Campylobacter and animal drug use. Int J Infect Dis 2004; 8: 190-2.

30.- Smith J L, Fratamico PM. Fluoroquinolone resistance in Campylobacter. J Food Prot 2010; 73: 1141-52.

31.- Unicomb L, Ferguson J, Riley TV, Collignon P Fluoroquinolone resistance in Campylobacter absent from isolates, Australia. Emerg Infect Dis 2003; 9: 1482-3.

32.- Engberg J, Neimann J, Nielsen E, Aarestrup F, Fussing V. Quinolone-resistant Campylobacter infections in Denmark: a risk factors and clinical consequences. Emerg Infect Dis 2004; 10: 1056-63.

33.- Funke G, Baumann R, Penner J, Altwegg M. Development of resistance to macrolide antibiotics in an AIDS patient treated with clarithromycin for Campylobacter jejuni diarrhea. Eur J Clin Microbiol Infect Dis 1994; 13: 612-5.

34.- Gibreel A, Taylor DE. Macrolide resistance in Campylobacter jejuni and Campylobacter coli. J Antimicrob Chemother 2006; 58: 243-55.

35.- Han J, Sahin O, Barton, Zhang Q. Key role of Mfd in the development of fluoroquinolone resistance in Campylobacter jejuni. En: http://www.plospathogens.org/article/ info\%3Adoi\%2F10.1371\%2Fjournal. ppat.1000083 (consultado el 14-01-2013)

36.- Cagliero C, Mouline C, Payot S, Cloeckaert A. Involvement of the CmeABC efflux pump in the macrolide resistance of Campylobacter coli. J Antimicrob Chemother 2005; 56 : 948-50.

37.- Habib I, Miller W, Uyttendaele M, Houf K, De Zutter L. Clonal population structure and antimicrobial resistance of Campylobacter jejuni in chicken meat from Belgium. Appl Environ Microbiol 2009; 75: 4264-72.

38.- Serichantalergs O, Pootong P, Dalsgaard A, Bodhidatta L, Guerry P, Tribble D, et al. PFGE, Lior serotype, and antimicrobial resistance patterns among Campylobacter jejuni isolated from travelers and US military personnel with acute diarrhea in Thailand, 1998-2003. Gut Pathog 2010; 2: 15-26.

39.- Saito S, Yatsuyanagi J, Harata S, Ito Y, Shinagawa K, Suzuki N, et al. Campylobacter jejuni isolated from retail poultry meat, bovine feces and bile, and human diarrheal samples in Japan: Comparison of serotypes and genotypes. FEMS Immunol Med Microbiol 2005; 45: 311-9. 